\title{
Taking Trajectory Based Operations to the Next Level: Management by Trajectory
}

\section{Alicia Fernandes}

Mosaic ATM, Inc.

April 26, 2018 


\section{MBT Concept Overview - Topics}

- Introduction and MBT overview

- Assumptions (operational environment)

- Key features of MBT

- Trajectories

- Assigned trajectory object

- Constraints

- Trajectory negotiation

- Benefit mechanisms

- Degraded modes/graceful degradation

- Summary and next steps 


\section{Introduction and MBT Overview}

\section{Current NAS}

Airspace users (AUs) plan flights without knowledge of all relevant constraints

Flight plan data is too sparse to support accurate trajectory prediction and synchronization

Operations that use open trajectories cause poor trajectory predictability

Not all control instructions are published, inhibiting trajectory synchronization

Poor trajectory predictability and synchronization inhibit strategic trajectory management

Insufficient reroute mechanisms cause backlogs during disruptive NAS events

\section{MBT}

NAS Constraint Service publishes all NAS and trajectory constraints

Assigned trajectory object includes the data needed for accurate, consistent 4DT predictions

Aircraft operate on closed trajectories to the extent possible

All control instructions are captured in the assigned trajectory and published

Improved trajectory predictability enables controllers to use strategic, closed clearances

Traffic managers apply constraints to amend trajectories as NAS events evolve

MBT enables a more flexible and responsive NAS that can take full advantage of available airspace and reduce delay 


\section{Operational Environment Assumptions}

\section{Aircraft Capabilities}

CPDLC and trajectory intent output

- Some aircraft may require manual entry of clearances into FMS

- Aircraft can provide intent via EFB and Air/Ground SWIM, or intent may come from Flight Operations Center (FOC)

All aircraft are capable of flying the assigned trajectory with known accuracy
Traffic Flow Management

- Time Based Management (TBM) is used in en route airspace

- GDPs and AFPs provide controlled arrival times (CTAs) rather than departure times (EDCTs)

- MIT restrictions that apply the same restriction across all aircraft pairs are eliminated

- When metering is not required, aircraft can be spaced using TBM or Interval Management

FOCs are capable of fully participating in MBT

- Aircraft not supported by an FOC can use automation and $3^{\text {rd }}$ party service providers

The NAS accommodates new aircraft classes and types of operations

- New aircraft classes may use MBT even in non-IFR portions of the NAS 


\section{Key Features of MBT}

- Trajectories

- Assigned trajectory object

- Constraints

- Trajectory negotiation 


\section{Trajectories and Assigned Trajectory Object}

Assigned Trajectory Object

\section{Business Trajectory}

Airspace user's

preferred trajectory

\section{Aircraft Capabilities}

Provide constraints on trajectories and clearances the aircraft can fly

\section{Assigned Trajectory}

\section{Trajectory Constraints} Minimum requirements that meet ATC and TFM needs

Trajectory Description

Additional data needed for trajectory prediction
Assigned trajectory object allows efficient exchange of all the data needed to predict the trajectory the aircraft will fly

FAA uses business trajectory for demand planning and identifies trajectory constraints

Airspace user and FAA negotiate an assigned trajectory that satisfies all constraints.

Airspace user updates aircraft intent throughout the flight. Intent may include details not in the assigned trajectory and may change without negotiation.
Airspace user's description of how the aircraft will fly in conformance with the assigned trajectory 


\section{Assigned Trajectory Object Examples}

\section{Assigned Trajectory Object}

\section{Assigned Trajectory}

FL310

Trajectory Constraint: AT OR ABOVE FL310, with no time constraint

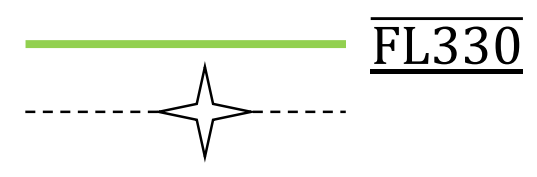

Trajectory Description: Aircraft will cross at FL330. Must negotiate to cross at FL350

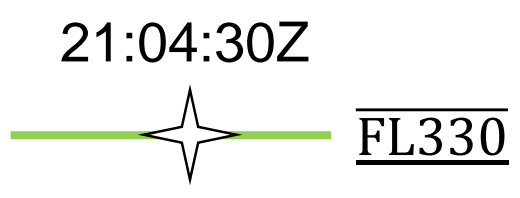

Aircraft Intent:

Aircraft will cross at 21:04:30Z.

No need to negotiate if crossing time changes 


\section{Assigned Trajectory Object - Description and Intent}

- Assigned Trajectory Object is a framework to handle different situations

Aircraft with minimal intent capabilities

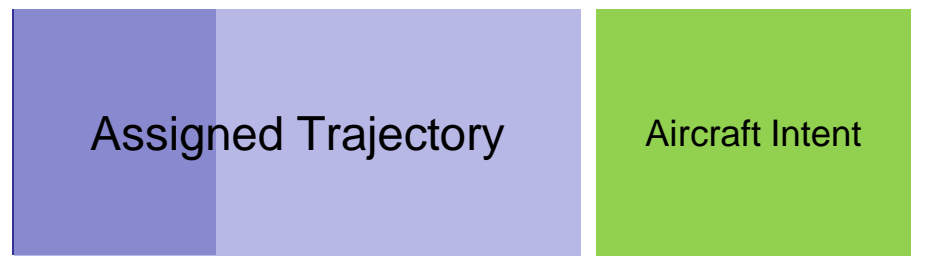

Aircraft provides detailed, accurate, timely intent

\section{Assigned Trajectory}

Aircraft Intent

- Predictability provided by detailed aircraft intent may support relaxing constraints in the assigned trajectory, increasing airspace user flexibility and decreasing negotiation requirement

\section{Research Question:}

What is the tradeoff between intent and trajectory constraints? 


\section{Constraints}

- NAS constraint: NAS element that affects the available assigned trajectories:

- ATM configuration information (e.g., SAA)

- Published procedure (e.g., STAR)

- Region of bad weather and resulting TMIs

- Strong turbulence or unfavorable winds

- Trajectory constraint: specific to a flight; trajectory must comply unless airspace user negotiates a change

- Assigned trajectory may reference the NAS constraints driving the trajectory constraints

- Supports identifying affected flights when a NAS constraint changes or is removed, capitalizing on opportunities to improve trajectory efficiency

NAS constraint: Metering in place for ATL arrivals $x^{*}$

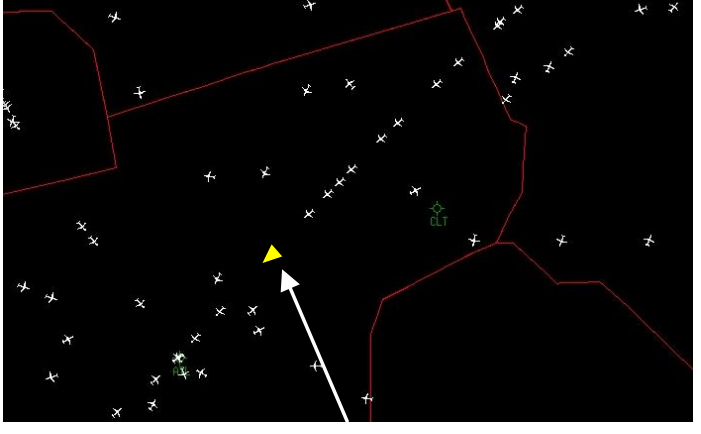

Trajectory constraint: STA at the meter fix

Can try to negotiate crossing time, but can only avoid metering by changing routes 


\section{MBT Trajectory Negotiation}

- MBT supports highly automated, complex trajectory negotiation, e.g.:

- When rejecting a trajectory, automation provides reason for rejection and constraints the proposed trajectory must meet

- E.g., "UNABLE TRAFFIC" vs. description of constraints/options

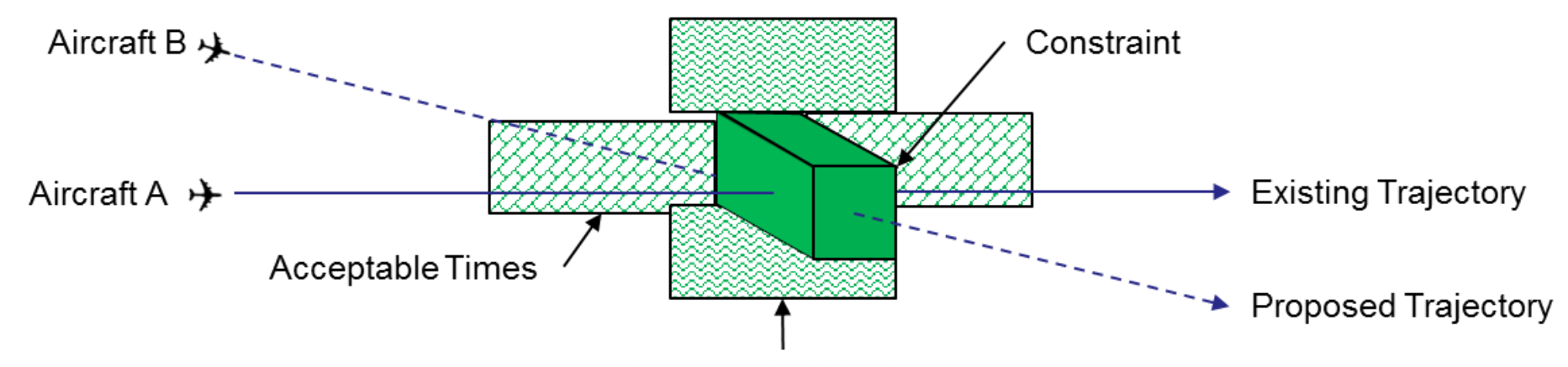

Acceptable Altitudes

- Offer airspace user a choice between two options, which is easily accomplished via voice

- Including aircraft capabilities in the assigned trajectory object is expected to improve negotiation efficiency

- FAA and airspace user propose "smarter" trajectories that are more likely to be accepted 


\section{Trajectory Negotiation Architecture}

Two separate but complementary systems:

- Negotiation takes place between negotiation automation on the ground (FOC and FAA) and onboard the flight deck (EFB)

- Upon reaching agreement, the negotiating controller issues a clearance using ATC automation

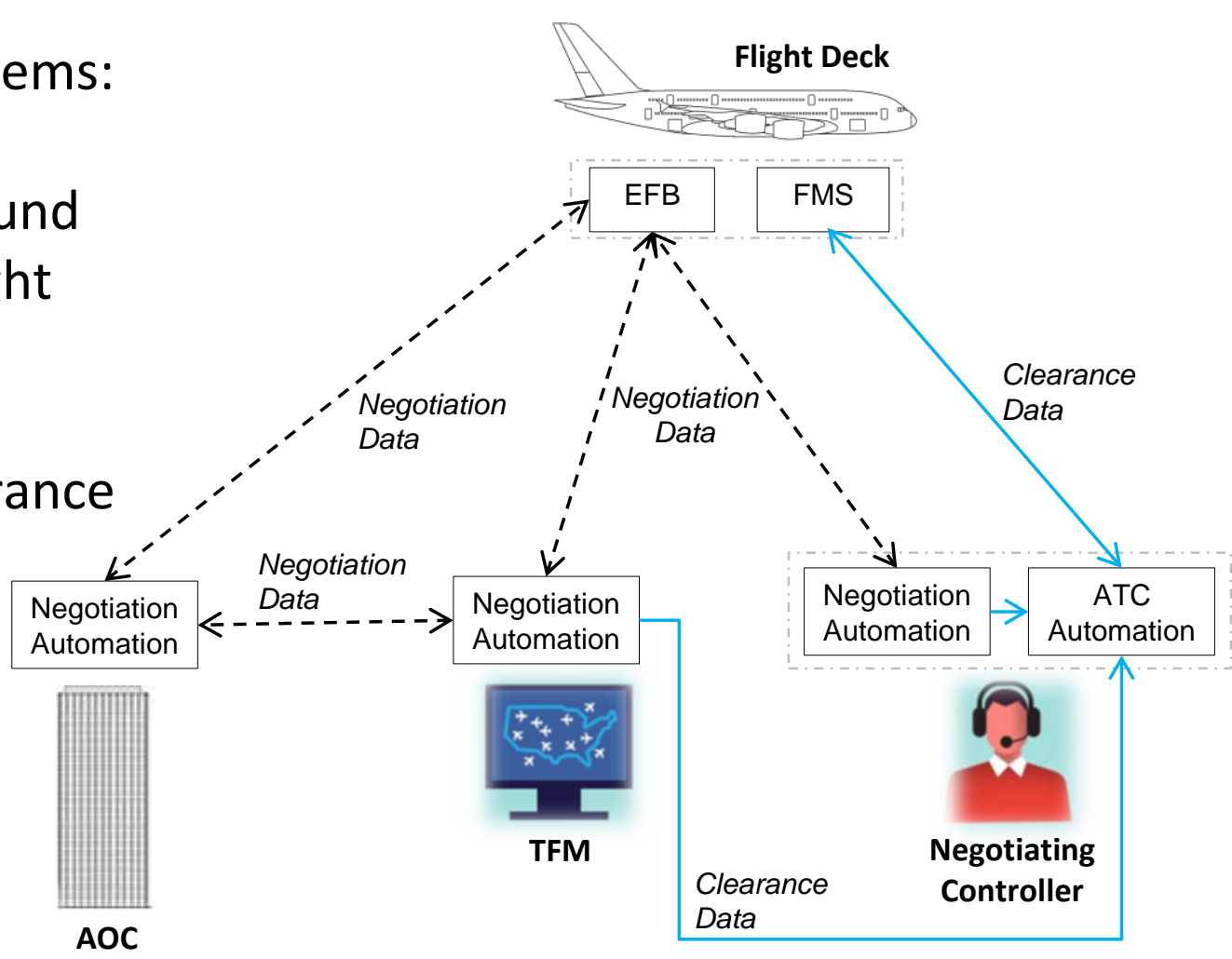




\section{Negotiation Interactions}

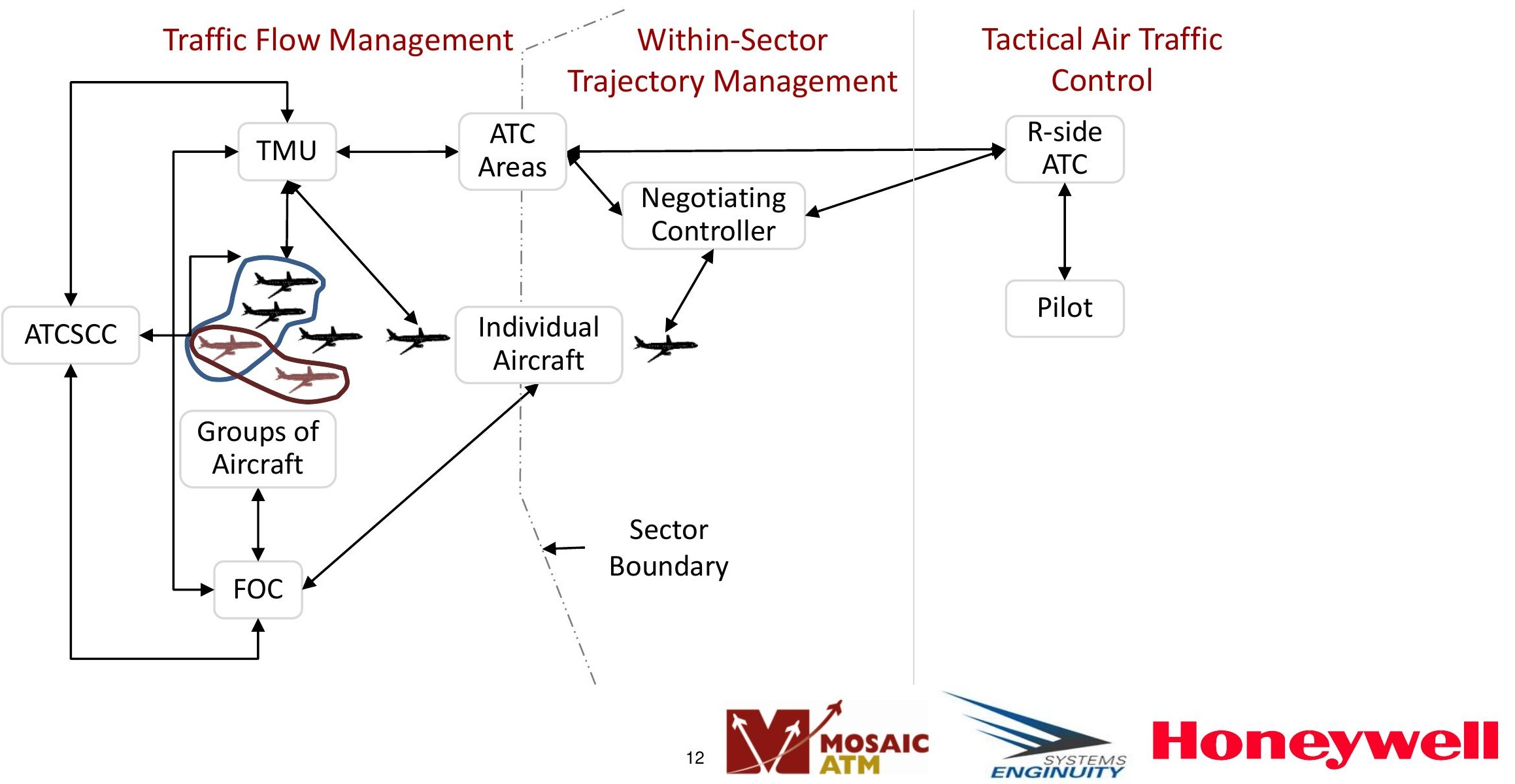




\section{Using the TOS in Trajectory Negotiation}

- Providing a TOS is optional, but it may reduce requirements for negotiation

- If a NAS constraint changes and FAA needs to reroute a flight, it will start negotiation from the TOS, if provided

- FAA could periodically evaluate the TOS to determine whether an alternate trajectory has become preferred

- Identifying a new preferred trajectory causes the FAA to process that trajectory as a requested trajectory to compute flight-specific constraints

- The resulting trajectory is presented to the airspace user for approval. If the airspace user accepts the trajectory, it becomes the new assigned trajectory.

- If the airspace user rejects the change, the alternative trajectory is removed from the TOS

- The first option in the TOS will be the currently assigned trajectory, unless the airspace user wishes to alter the assigned trajectory 
- Efficiency

- Safety

- Access to the NAS 


\section{MBT Benefit Mechanisms - Efficiency}

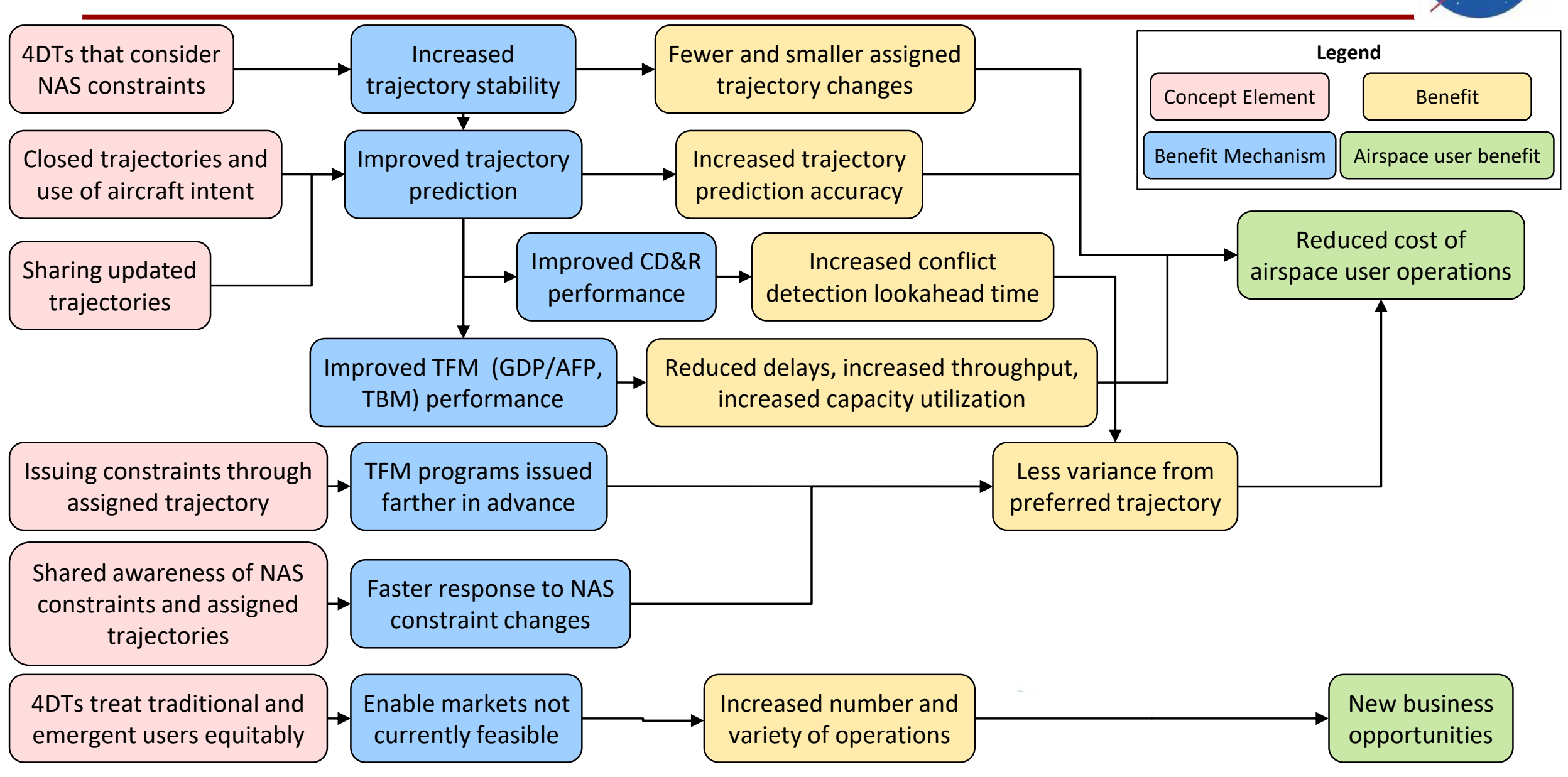




\section{MBT Benefit Mechanisms - Safety}

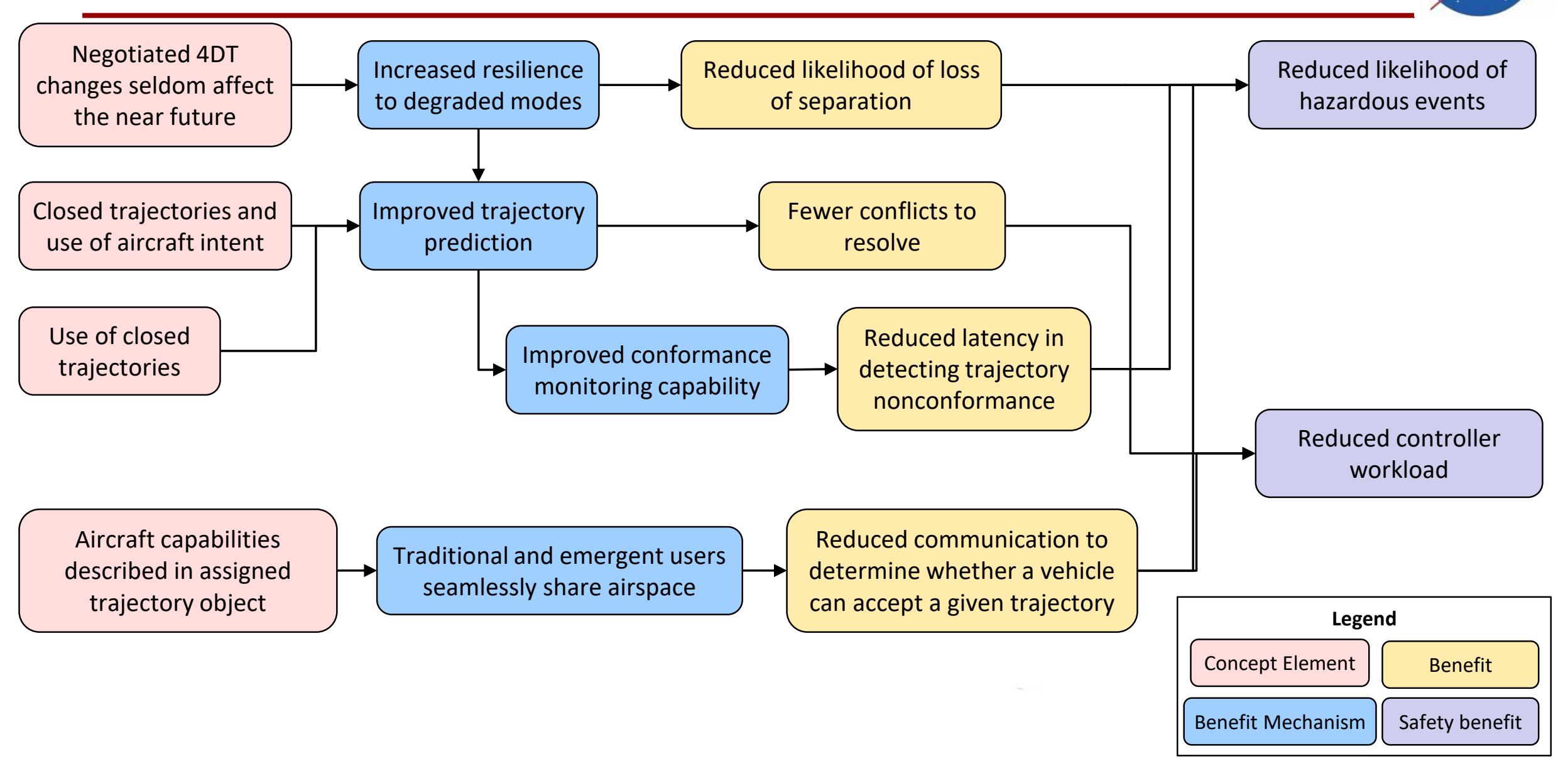




\section{MBT Benefit Mechanisms - Access to the NAS}

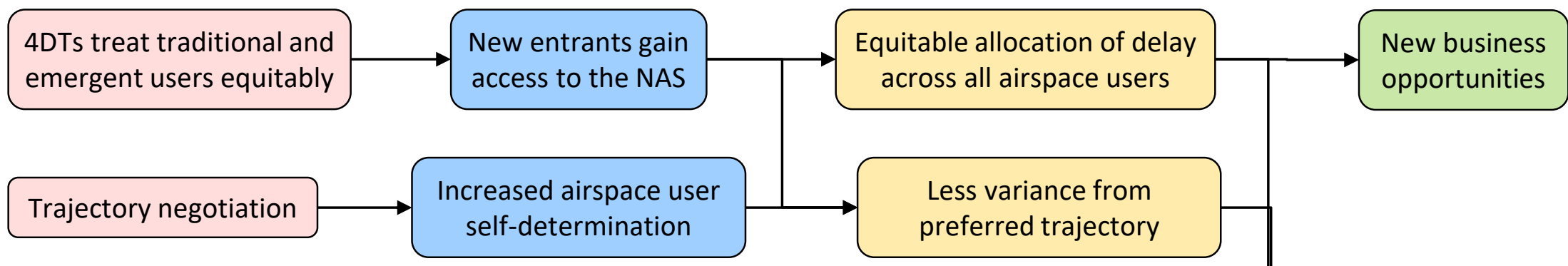

Airspace users that adopt new capabilities better able to negotiate preferable trajectories
Encourages equipage that further enables efficiency and safety benefits
Increased participation in trajectory negotiation
Reduced cost of airspace user operations

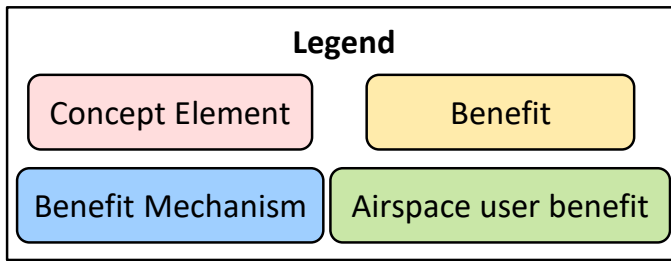




\section{MBT Concept Summary (1/2)}

- Assigned trajectory from flight's current state to its destination composed of:

- Minimal set of trajectory constraints to achieve safety and efficiency goals

- Trajectory description so the assigned trajectory is a complete trajectory when few trajectory constraints are required

- All aircraft follow their assigned trajectories unless they negotiate a revised trajectory

- All airspace users provide and maintain trajectory intent and aircraft capability info

- Aircraft intent may contain details such as ETAs at waypoints that do not have time constraints in the assigned trajectory

- Intent can change freely without negotiation, as long as it conforms to the assigned trajectory

- Together, the assigned trajectory and aircraft intent enable accurate prediction of the 4DT that the aircraft will fly

Management by Trajectory achieves the FAA's goal of Trajectory Based Operations and supports integration of emerging vehicle classes and business models into the NAS 


\section{MBT Concept Summary (2/2)}

- NAS Constraint Service gathers and publishes information about all known NAS constraints

- Assigned trajectory references NAS constraints driving the trajectory constraints

- Facilitates identifying aircraft affected by changes to (or removal of) NAS constraints

- Uncertainty and disruptions are handled by modifying the assigned trajectory as far in advance as possible

- Allows changes to be negotiated and communicated as assigned trajectory amendments and not tactical control actions

- MBT enables more accurate trajectory predictions, leading to:

- Improved ATM performance and robustness to off-nominal conditions

- Increased flexibility and operational efficiency

MBT reduces impediments to emerging classes of airspace users accessing the NAS 


\section{MBT Next Steps}

- Simulation to quantify:

- Safety, efficiency, and performance effects and requirements

- Required level of trajectory predictability (and stability) to achieve safety and efficiency improvements

- MBT impact on trajectory predictability and stability

- Tradeoffs between trajectory constraints, quality of trajectory intent, and airspace user flexibility

- Additional concept engineering

- More detailed requirements for the assigned trajectory object and trajectory negotiation process

- Prototype automation and decision support tools to validate roles and responsibilities

- Detailed transition plan from the current environment to the full MBT vision 


\section{Backups}




\section{Trajectories in MBT (1)}

- Assigned Trajectory - the 4DT the airspace user agrees to fly

- Represents a minimal set of requirements to meet FAA objectives and enable prediction of the aircraft's trajectory; constructed in two parts:

- Trajectory constraints: the minimum set of requirements that achieve ATM needs (i.e., conflict avoidance) and TFM needs

- As the minimum required set, may not fully describe where and when the aircraft will fly

- Trajectory description: provides the additional information necessary to support trajectory prediction

- Result of negotiation between airspace user and FAA

- Initially created pre-departure; updated as needed until flight reaches destination

- The flight must conform to everything in the assigned trajectory, or renegotiate 
- Actual Trajectory - the 4DT actually flown (and taxied) by an aircraft

- Predicted Trajectory - a 4DT the aircraft is predicted to follow

- Different systems may compute predictions for their own purposes

- Predictions and data used in predictions are shared

- Business Trajectory - a 4DT that the operator wants to fly or provides as the requested trajectory

- Starting point for negotiation of assigned trajectory

- May change over the course of flight 


\section{Moving Forward}

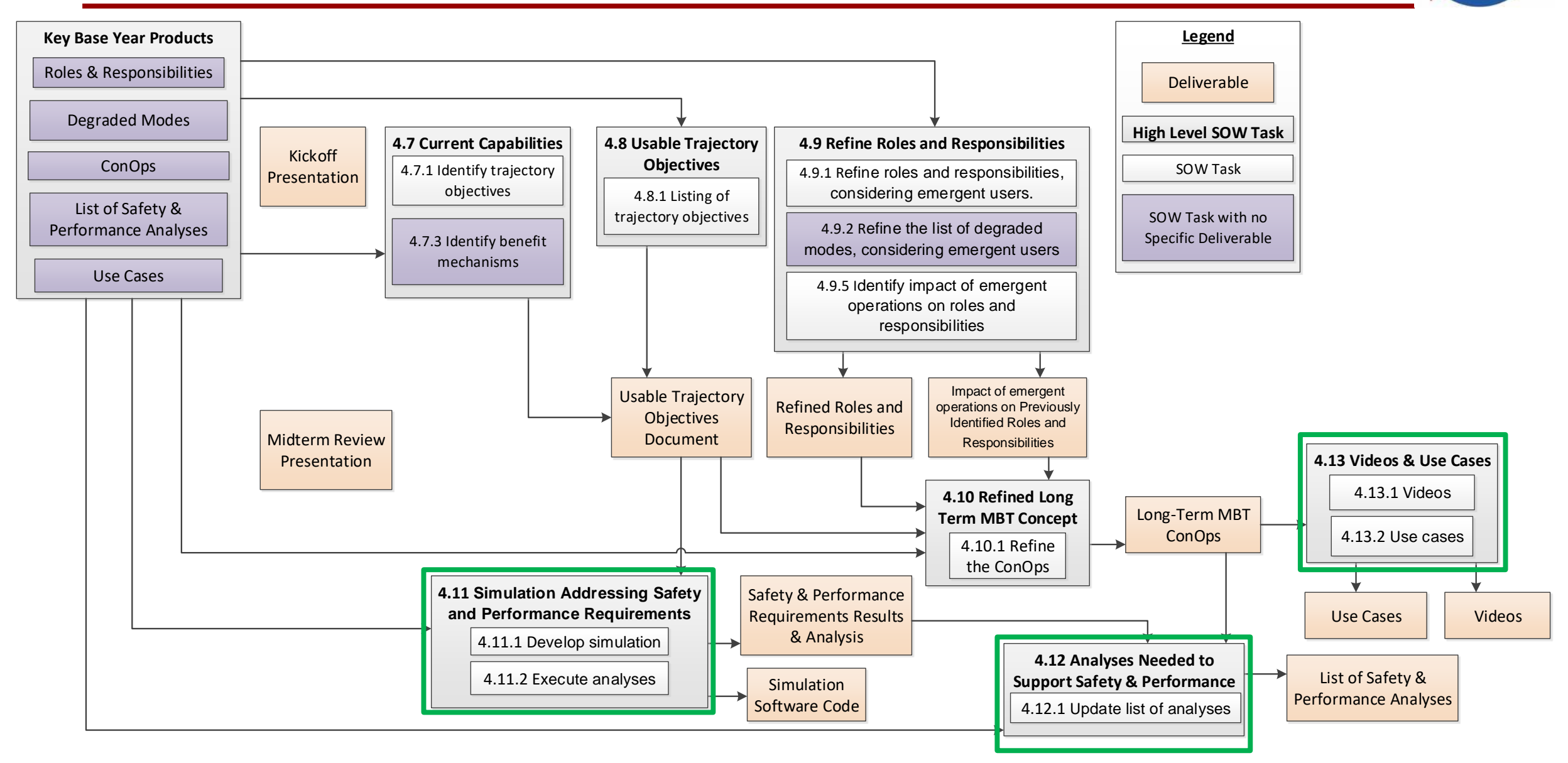




\section{MBT Project Schedule}

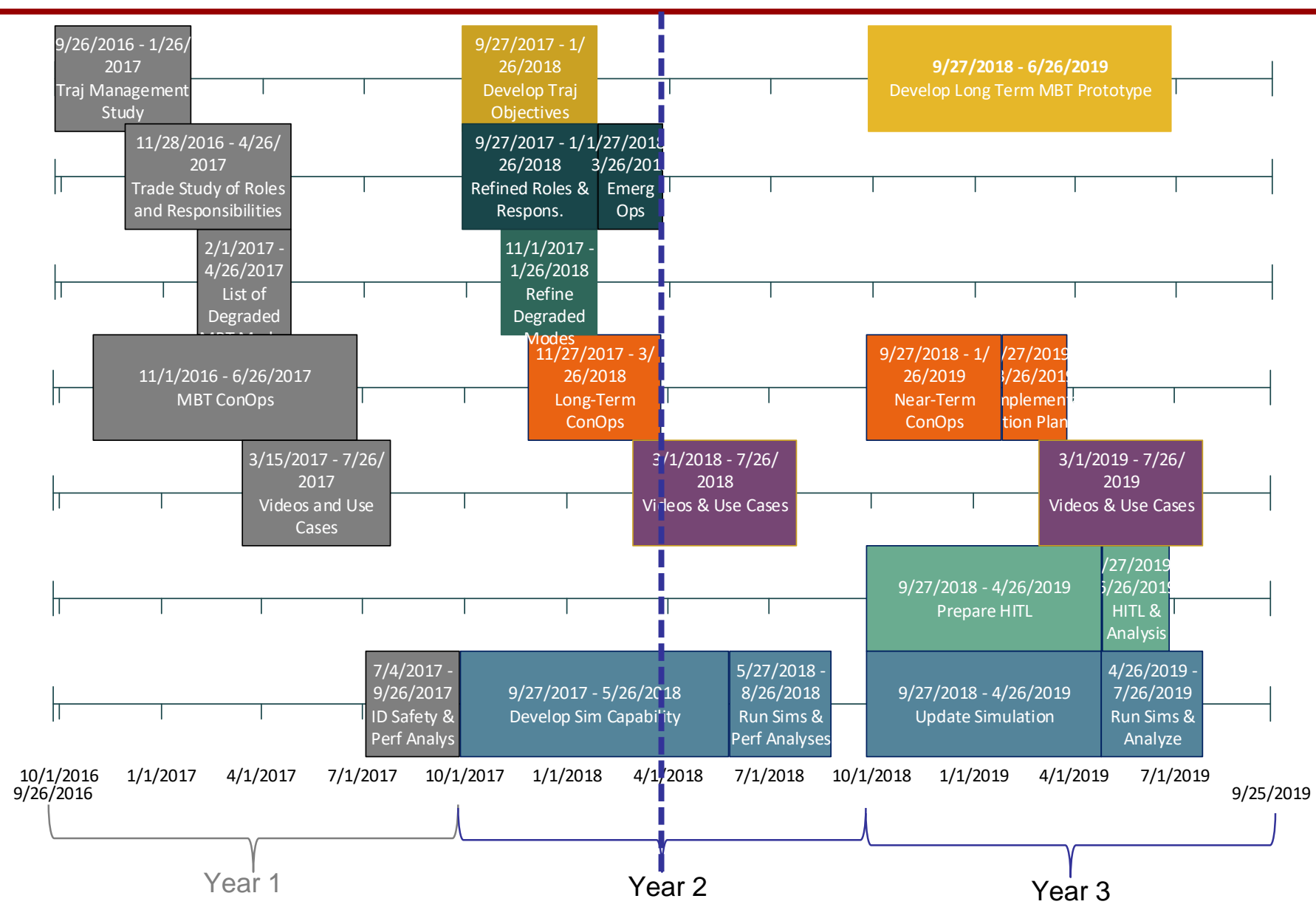




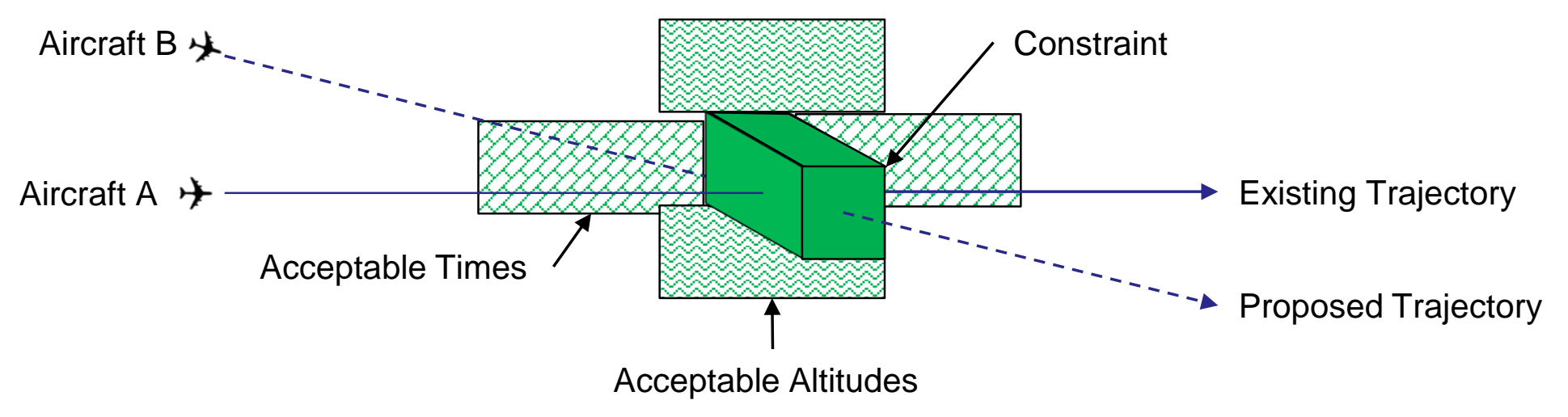

